\title{
Aggregate interlocking of open-graded friction courses with compaction effort
}

\author{
W.K. Mampearachchi", S.R. Masakorala and K. Umasangar \\ Department of Civil Engineering, Faculty of Engineering, University of Moratuwa, Katubedda, Moratuwa.
}

Submitted: 14 February 2018; Revised: 17 July 2018; Accepted: 26 October 2018

\begin{abstract}
Densification of open-graded friction course (OGFC) makes it inefficient, with the reduction of air void content due to aggregate breakage by over compaction efforts. Therefore, it is important to identify the optimum compaction effort to accomplish the most stable aggregate on aggregate contact of internal aggregate structure with increasing compaction. This paper presents the optimum number of gyrations that would be needed for aggregate locking. Samples were prepared with various gradations, binder contents and compaction levels to compare different combinations of these conditions on the optimum compaction. The aggregate interlocking point was found using two methods; (a) by observing height variation of the sample and (b) by calculating the gradient of height variation curve generated by superpave gyratory compactor (SGC). According to method (a) aggregate interlocking point was identified and it was observed that compaction effort increases with coarser mixtures. As per method (b), the calculated interlocking point, which was independent from individual variations was found proximate to 50 gyrations as an average among all samples under various gradations, binder contents and compaction levels. Both methods provided compaction results greater than $85 \%$ of the maximum theoretical density at the interlocking point. The results concluded that the gradient calculation method can be adopted successfully as an unbiased method to find the aggregate interlocking point of OGFC samples to determine the optimum compaction level.
\end{abstract}

Keywords: Aggregate interlocking, OGFC, optimum compaction, SGC.

\section{INTRODUCTION}

As stated by the Federal Highway Administration (FHWA) 'open-graded friction course (OGFC) is a thin, open-graded asphalt mixture placed on the dense graded pavement' formulated for improved road safety during wet weather conditions by removing surface runoff quickly through an interconnected porous structure (Smit \& Prozzi, 2013; Techbrief, 2015). Thus, it reduces spray and splash due to surface ponding (Liu et al., 2010), hydroplaning and noise generation (Liu et al., 2016) while increasing wet weather frictional resistance and visibility. Therefore, it is especially beneficial for roads with high operating speeds. In different countries, OGFC is referred to in various ways: permeable friction courses (PFC-US), porous asphalt, porous european mixes (PEMEU) or stone matrix asphalt (SMA-India) (Chandra \& Kumar, 2016). Since 1950's OGFC has been used in the United States and the FHWA introduced a mix design initially in 1974. It was unsuccessful in the early stages as the pavement lifetime was limited to about $8-12$ years (Liu et al., 2010) due to the low integrity of pavement and the clogging of the porous structure by fine particles. After about 30 years of introducing, only 14 out of the 50 states had adopted OGFC by 2009 (Putman, 2012).

The most important task of OGFC is compacting without causing an adverse impact on permeability through voids content (Choubane et al., 1997). Permeability increases with the increase of interconnected voids in porous structure. As a comparison, the content of air voids were $4-6 \%$ in dense-graded pavement after compaction, which is much lower than that in OGFC (16-22\%) recommended by the FHWA (Techbrief, 2015). In the field, OGFC compaction is usually

*Corresponding author: (wasanthak@uom.lk; (iD https://orcid.org/0000-0002-8762-1234) 
performed using static wheeled rollers and tandem rollers of 8-10 tons (Techbrief, 2015). Maintaining the content of air voids during construction directly depends upon the compaction effort, method, gradation, binder content, aggregate properties, and nominal maximum aggregate size of the mixture. To perform field compaction under laboratory conditions, mainly, the Marshall method and the Superpave method are successfully used (Kandhal \& Mallick, 1999). However, superpave gyratory compactor (SGC) is considered as a better simulation of specimens very close to the field compacted sample properties. One possible reason is, its simultaneous impacts of vertical compression and rotation about vertical axis, while the Marshall hammer provides only a vertical impact. In addition, primary SGC operating parameters such as internal angle, rotation of gyrations, applied pressure and number of gyrations may also affect the resultant compaction (Techbrief, 2010).

The main purpose of OGFC compaction is to satisfy the required air content with a stable internal aggregate structure that would be determined by the aggregate interlocking point. Consequently, efficient drainage and resistance to structural deformation ultimately causes the long-lasting serviceability of the pavement (Cindy, 2007). This paper discusses the variation of minimum compaction energy required (number of gyrations) to reach this optimum compaction.

Aggregate interlocking has been identified as an important characteristic in OGFC due to its inherent porous structure (Vavrik, 2000; Birgisson et al., 2006). Varvik (2000) proposed a method to optimise aggregate interlocking by combining different coarse and fine aggregate gradations by volume. The results were based on mix design properties following a complex and lengthy procedure. In the same study, locking point of SGC-compacted samples were defined at the point, which began to indicate resistance to compaction on the generated density curve. Accordingly, Virvik (2000) concluded that the gradation has an effect on interlocking point. When the design unit weight increases by increasing the coarse aggregate percentage, the number of gyrations to achieve the interlocking point increases. This behaviour is due to the demanding of a higher unit weight by increasing coarser aggregates, which are difficult to rotate and displace rather than fine particles.

In 2015, the Varvik method was developed by Birgisson et al. (2006) to identify the interlocking point of SGC-compacted samples using the rate of gradient of the generated compaction curve. Using regression analysis, they presented the interlocking point by the gradient of log compaction curve. It was identified at two similar gradients preceded by two consecutive gyrations with similar gradients on the compaction curve. The locking point was found for limestone and granite samples as 46 and 56 gyrations, respectively. The gradation after compaction indicated further reduction of aggregate sizes due to the breakdown of limestone, which occurred at relatively low compaction. Therefore, the investigation proposed 50 gyrations of optimum compaction for OGFC.

Similarly, many studies and specifications have recommended 50 gyrations equivalent to achieve optimum compaction (Kandhal \& Mallick, 1999; Vavrik, 2000; Watson et al., 2002; Alvarez et al., 2007; NCAT, 2000). Kandhal and Mallick (1999) compared SGCcompacted samples of different gyration levels with field samples. It was found that about 50 gyrations simulate the field compaction with $18 \%$ air voids content. In a similar study, Watson et al. (2002) suggested 50 gyrations among three gyration levels. 30, 45 and 60 gyrations of SGC and 25 and 50 Marshall blows were used in the study due to; (1) equal bulk specific gravity $\left(\mathrm{G}_{\mathrm{mb}}\right)$ between 50 Marshall blows and 50 gyrations, (2) low aggregate breakdown in 25 Marshall blows when compared to 60 gyrations; (3) satisfying aggregate-on-aggregate contact criteria (based on coarse aggregate content $>4.75 \mathrm{~mm}$ ); and (4) low aggregate loss in Canterbro test. Another research by the Texas Transportation Institute reproduced HMA samples with the required air voids content with 12-15 gyrations initially $\left(\mathrm{N}_{\text {initial }}\right)$, to achieve aggregate-onaggregate contact condition with at least $70 \%$ of total compaction. However, air void content and permeability reduction during 15 and 50 gyrations were not much significant and gave approximately $30 \%$ and $10 \%$, respectively (Alvarez et al., 2007).

Changing the internal aggregate structure was described by Sefidmazgi (2011) relevant to aggregate gradation, binder properties, compaction method, mixture temperature and compaction effort, using digital imaging. Gradation graphs of different asphalt samples analysed using Weibull distribution gave the maximum interlocking for the dense-graded mixture with higher coarse content (46\% passing $4.75 \mathrm{~mm}$ ) and gap-graded sample without $4.75 \mathrm{~mm}$ size aggregate (Weibull distribution was a function of fine percentage and grade, which could be gap-graded or dense-graded).

According to literature, achieving stability of the asphalt mixture with specified properties such as the air void content and resistance to deformation is difficult to accomplish in the field without predetermined conditions. 
However, many studies have been conducted on aggregate packing based on various concepts by varying aggregate types and coarse aggregate percentage (Vavrik, 2000; Watson et al., 2002; Sefidmazgi, 2011) for HMA. However, due to significant variations of OGFC gradations and volumetric properties, further investigations are needed to establish a strong procedure to evaluate optimum compaction through the interlocking concept.

The objective of this research was to find the minimum number of gyrations required for optimum compaction of OGFC with different gradations under laboratory conditions by SGC and regression analysis. The term optimum compaction is defined as the level of compaction before the starting of the aggregate interlocking without disturbing the internal stability of the aggregate structure.

\section{METHODOLOGY}

Test samples were prepared for SGC according to the American Association of State Highway and Transportation Officials (AASHTO) specification (WDOT, 2016) and HMA mixture designs following the superpave aggregate gradations and binder contents within the range recommended by FHWA (Techbrief, 2015) for OGFC as given in Table 1. The selected aggregate gradations were designed by Gunaratne and Pernia (2015) for the determination of the optimum binder content of OGFC samples. Coarse aggregate percentages were considered by the percentage of passing through $4.75 \mathrm{~mm}$ sieve (No. 4). Granite aggregate and 60/70 penetration grade bitumen were selected for the mixtures. Therefore, G1 of the selected gradations is coarser than G3. Binder content was taken as $5.3 \%$ for S1-G1, S2-G2

Table 1: Aggregate gradation for samples

\begin{tabular}{lcccc}
\hline Sieve size & $\begin{array}{c}\text { FHWA } \\
(12.5 \mathrm{~mm})\end{array}$ & $\mathrm{G} 1$ & $\begin{array}{c}\text { \% Passing } \\
\text { G2 }\end{array}$ & G3 \\
\hline 19 & 100 & 100 & 100 & 100 \\
12.5 & $85-100$ & 96 & 95 & 96 \\
9.5 & $55-75$ & 71 & 74 & 70 \\
4.75 & $15-25$ & 15 & 20 & 23 \\
2.36 & $5-10$ & 8 & 8 & 10 \\
1.18 & & 6 & 6 & 5 \\
0.6 & & 5 & 4 & 4 \\
0.3 & & 4 & 4 & 3 \\
0.15 & $2-4$ & 3 & 4 & 3 \\
0.075 & & 2.3 & 3.4 & 2.5 \\
\hline
\end{tabular}

FHWA: Federal Highway Administration and S3-G3 and $5.5 \%$ for one sample, S4-G3. Another three samples of the same gradation (S5-G2, S6-G2 and S7-G2) were prepared using $5.8 \%$ binder content, which was the minimum recommended by FWHA for OGFC (9.5 mm) (Techbrief, 2015).

Samples were compacted using SGC. Table 2 shows the data provided for Gyratory compactor software V 3.6.1. The SGC parameters (speed, internal angle and compaction stress) were not changed, while compacting for all the samples. Only the sample weight, binder content and number of gyrations were changed during sample preparation. The mold selected was $100 \mathrm{~mm}$ in diameter, except for S6-G2 (150 mm diameter). Maximum theoretical density $\left(\mathrm{G}_{\mathrm{mm}}\right)$ and bulk density $\left(\mathrm{G}_{\mathrm{mb}}\right)$ were estimated initially (Table 2$)$ as a reference for future calculations.

During compaction, sample height was continuously measured using the displacement transducer by compactor. Afterwards, the software calculated the percentage change of $\mathrm{G}_{\mathrm{mm}}\left(\% \mathrm{G}_{\mathrm{mm}}\right)$ for each gyration with respect to the provided $\mathrm{G}_{\mathrm{mm}}$. Then the $\mathrm{SGC}$ generated the gyrations versus density curve and the gyrations versus height curve for the given mixtures (Gyratory compactor operating manual).

Table 2: Data for gyratory compactor software V 3.6.1 (for S2-G2)

\begin{tabular}{lc}
\hline Specification & AASHTO T312-09 \\
\hline Mold diameter $(\mathrm{mm})$ & 100 \\
Mass of material $(\mathrm{g})$ & 2070 \\
Mold spacer height $(\mathrm{mm})$ & 0 \\
No. of gyrations & 50 \\
Speed (Rev/min) & 30 \\
Internal angle (Deg.) & 1.16 \\
Compaction stress $(\mathrm{KPa})$ & 600 \\
$\mathrm{G}_{\mathrm{mm}}$ & 1.302 \\
$\mathrm{G}_{\mathrm{mb}}$ & 1.235 \\
Binder percentage $\%$ & 5.3 \\
\hline
\end{tabular}

\section{RESULTS AND DISCUSSION}

When an asphalt mixture densifies, the aggregates tend to acquire internal stability through internal aggregate structure properties such as aggregate contact area and aggregate orientation. For instance, the number of particles that were contacted (contacted area) indicates the interconnectivity of the internal aggregate structure, which is important in load transferring. Further, aggregate orientation normal to pavement surface (loading surface) 
reduces deformations due to traffic loading. Eventually, aggregate particles form a stable interconnected porous structure with a higher content of air voids (permeability). This state is known as aggregate interlocking with maximum aggregate-on-aggregate contact. Coarse aggregate content mainly involves the angularity and surface roughness for aggregate packing density (Vavrik, 2000; Mahboub, 2004). Hence, higher coarse content in OGFC would satisfy the permeability requirement.

However, for all the samples, the density rapidly increased at the initial stage and reduced after a certain number of gyrations due to internal resistance to compaction due to aggregate interlocking. When the aggregate structure begins to resist compaction, excess energy beyond the resistive point will be absorbed by the aggregate, which leads to cracking and breaking down of particles.

As crushing aggregates fill the interconnected air voids in OGFC mixtures, low permeability could be observed with excessive compaction according to the literature (Watson et al., 2002; Alvarez et al., 2007). Therefore, optimum compaction is considered to be achieved below the interlocking point.

The software-generated height and density variation curves of the samples with respect to the number of gyrations indicated how the samples react when increasing the compaction effort. Height variation curves and compaction variation curves are shown in Figures 1 (a) and (b) for S1-G1, S2-G2 and S3-G3 samples. The figures illustrate that the sample height is inversely proportional to the compaction rate. Due to the high porous nature of OGFC, sample density increased rapidly in the beginning and then the rate of compaction decreased significantly beyond the initial stage of compacting. Finally, it changed slightly. This initial compaction $\left(\mathrm{N}_{\text {initial }}\right)$ was not considered in the experiment.

Subsequently, a resistance to compaction was observed in several samples compacted beyond 40 gyrations. As shown in Figure 2, height variation at some locations was not observed significantly or completely with the compaction energy provided. Therefore, an assumption was made that the aggregate in the sample began to breakdown to facilitate further compaction. As a result of over compaction, disturbances to aggregate-on-aggregate contact and clogging of porous aggregate skeleton occur beyond the point mentioned above. Most importantly this situation should be avoided during OGFC compaction in the field for better functionality.

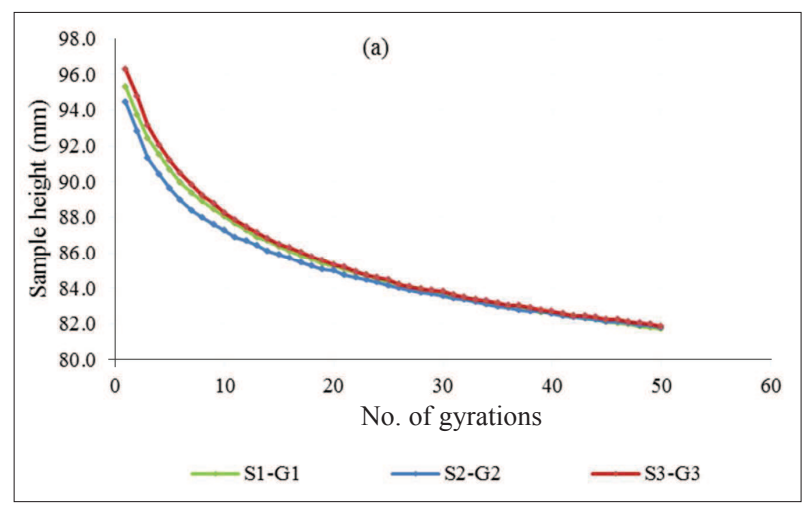

Figure 1(a): Gyrations vs height

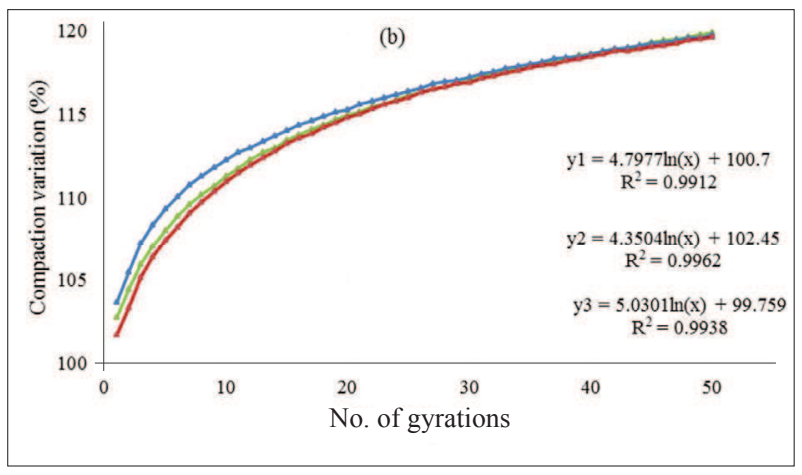

Figure 1(b): Gyrations $v s$ compaction

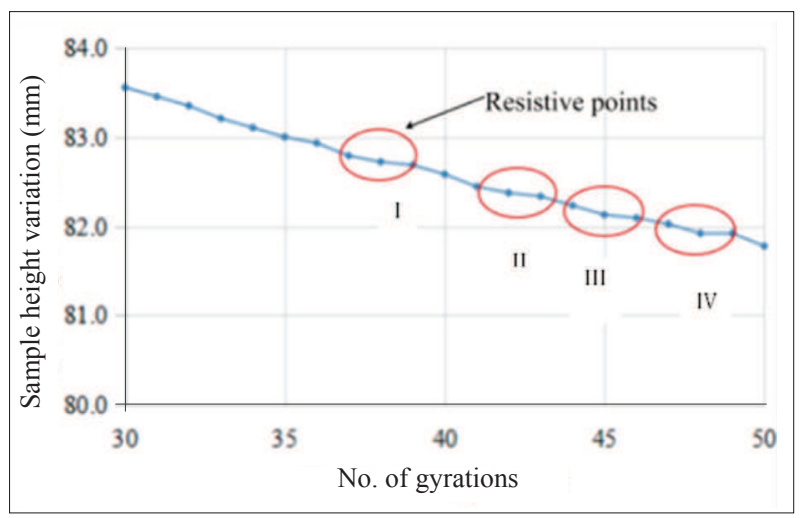

Figure 2: Sample height variation with number of gyrations (for S2-G2)

To identify the aggregate interlocking point of the given samples the maximum density that could be achieved without aggregate breakdown should be known. The number of gyrations related to the above condition can be considered as the optimum compaction required for the OGFC sample without causing disturbances to the internal aggregate skeleton. 
From the height variation curve, the gyration before two successive similar heights was considered as the aggregate interlocking point, when the sample heights were rounded to one decimal place (Table 3). These similar consecutive heights indicate the resistivity of the aggregate to compact further with the provided impact. In addition, as this height variation is inversely proportional to the sample density, the identified resistive point can be considered as an interlocking point or maximum aggregate-on-aggregate contact for the particular sample.

Table 3: Interlocking point according to sample height (for S2-G2)

\begin{tabular}{cc}
\hline Gyrations & Sample height $(\mathrm{mm})$ \\
\hline 33 & 83.2 \\
34 & 83.1 \\
35 & 83.0 \\
36 & 82.9 \\
37 & 82.8 \\
38 & 82.7 \\
39 & 82.7 \\
40 & 82.6 \\
\hline
\end{tabular}

Table 4: Comparison of interlocking point with coarse content

\begin{tabular}{lcccc}
\hline Sample ID & $\begin{array}{c}\text { \% Passing } \\
4.75 \mathrm{~mm}\end{array}$ & $\begin{array}{c}\text { Total } \\
\text { gyrations }\end{array}$ & $\begin{array}{c}\text { Method } \\
\text { (a) }\end{array}$ & $\begin{array}{c}\text { Method } \\
\text { (b) }\end{array}$ \\
\hline S1-G1 & 15 & 50 & 45 & 44 \\
S2-G2 & 20 & 50 & 37 & 43 \\
S3-G3 & 23 & 50 & 34 & 44 \\
\hline
\end{tabular}

Following this concept, aggregate interlocking points for S1-G1, S2-G2 and S3-G3 samples were identified as shown in Table 4 [indicated as method (a)]. In graphical presentation of comparison of interlocking point with percentage passing $4.75 \mathrm{~mm}$, it is clearly illustrated that the higher coarse aggregate content needed higher compaction effort to reach the aggregate interlocking point (Figure 3). As mentioned above, interlocking points for samples (S5-G2 and S6-G2) compacted with 40 gyrations were not observed due to the given compaction being insufficient to reach the stable aggregate-on-aggregate contact condition.

However, according to the SGC generated gyrations versus height graph, four points were identified with significant disturbance to densification of $\mathrm{S} 2-\mathrm{G} 2$ as

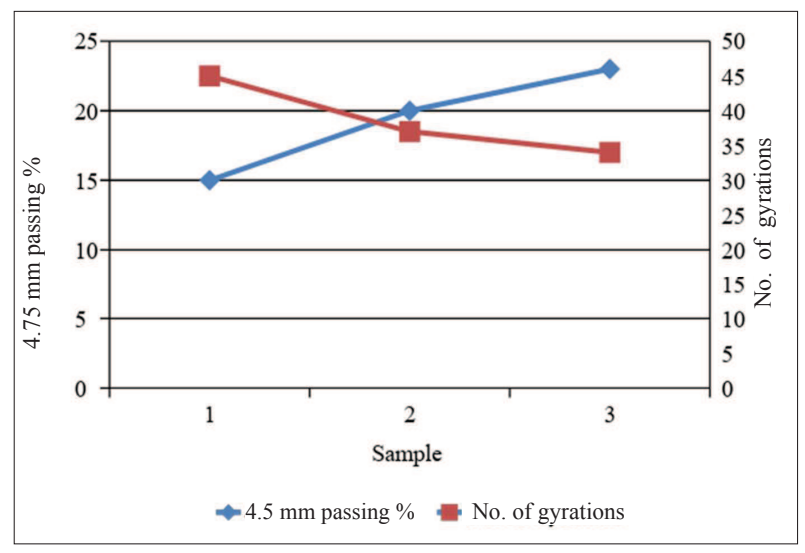

Figure 3: Comparison of interlocking point with percentage passing $4.75 \mathrm{~mm}$

shown in Figure 2. Although points I, II and III show a significant resistance to compaction, point IV showed a totally unchanged compaction. Therefore, it was difficult to define an exact optimum point. In such situations, an analytical method based on the provided data by SGC is better to be implemented when identifying the interlocking point or the number of gyrations needed for optimum compaction.

Height variation of the sample is a direct measurement given by the SGC, which is used to calculate other output parameters. Hence, a relationship between the number of gyrations versus sample height (equation 1) was improved as given below;

$$
y=-m \ln (x)+c
$$

where, $y$ and $x$ are sample height and the number of gyrations, respectively and $m$ is the gradient of the $\log$ curve. Then, the rate of height for each gyration was determined by second order differential equation (equation 2) derived from equation (1) for better illustration of the change of density.

$\frac{d^{2} y}{d x^{2}}=\frac{m}{x^{2}}$

Then, following the same procedure, number of gyrations for two consecutive similar gradients was considered as the aggregate interlocking point as shown in Table 5.

Accordingly, interlocking point for S1-G1, S2-G2 and S3-G3 was given at average 44 gyrations (Table 4). Therefore, the aggregate gradation effect was not observed with the following results unlike in 
previous occasions; the calculated gradients were only based on the gradient of logarithmic height curve (in equation 1) and number of gyrations. And it was due to the lesser sensitivity of the resultant graph to the individual variation of compaction. It illustrates the rate of compaction theoretically and gives equal values when an insignificant rate of gradient is on the curve. One of the major advantages of this method is the prediction of the interlocking point without achieving it when equation (1) is available. However, effect of the compaction level and binder content was compared further with methods (a) and (b).

Table 5: Interlocking point according to gradient calculation for sample (S2-G2)

\begin{tabular}{cc}
\hline Gyrations & Variation of gradient \\
\hline 39 & 0.0022 \\
40 & 0.0021 \\
41 & 0.0020 \\
42 & 0.0019 \\
\hline 43 & 0.0018 \\
44 & 0.0017 \\
45 & 0.0017 \\
46 & 0.0016 \\
47 & 0.0015 \\
\hline
\end{tabular}

Consequently, S5-G2, S6-G2 and S7-G2 samples with $5.8 \%$ binder content and G2 gradation for 40 and 80 gyrations compaction levels indicated an interlocking point at a higher number of gyrations as 54, 55 and 54 (average 54) by method (b). As previously [method (a)], an interlocking point was identified only for one sample, S7-G2 at 58 gyrations level. The other two samples were considered not to have reached the optimum compaction level. However, no relationship was observed between the final compaction level and the aggregate interlocking points.

The proposed method (b) to find interlocking points using equation (2) was advantageous, as calculations were only based on the gradient of the height curve in log scale and the number of gyrations. Thus, the interlocking point can be calculated when the gradient is known. Calculated gradients were theoretical and not sensitive to individual variations of compaction. However, deciding the locking point with a height variation curve [method (a)] is subjective to visual observations. Both methods were compared under different mix designs.
Further, variation of interlocking points with respect to binder content, neglecting the gradation effect and the number of gyrations to achieve total compaction, is shown in Figure 4 (for all samples, S1-G1 to S7-G2). A strong correlation between the two parameters; binder content in mixtures and gyrations, was observed by method (b). According to the graph, increasing the binder content indicated higher energy requirement (number of gyrations). One possible reason is that the tenderness of the mixture tend to make a more compacted mixture without aggregate breaking upon increasing the binder content. Accordingly, locking point tends to increase when increasing the binder content and requires a higher compaction effort.

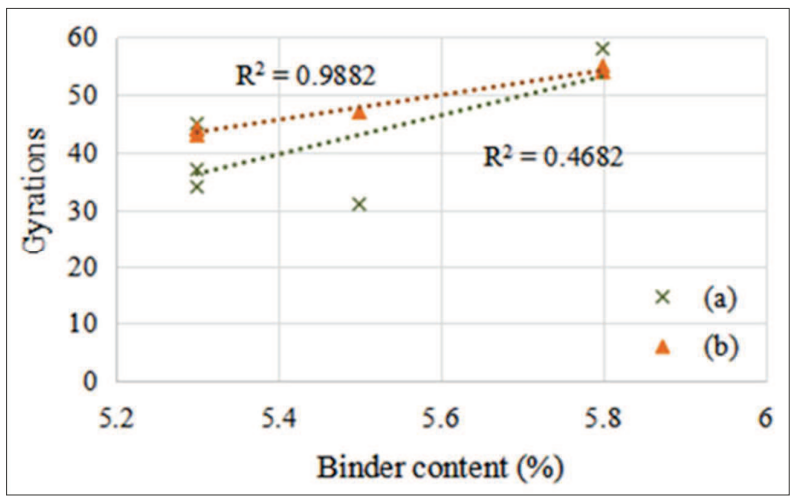

Figure 4: Binder content $v s$ number of gyrations

In summary, the selected aggregate gradations show a maximum of $8 \%$ variation of coarse content, from $77 \%$ to $85 \%$ (greater than $4.57 \mathrm{~mm}$ size) and the binder content varies from $5.3 \%$ to $5.8 \%$. Samples were compacted under different gyration levels to observe the aggregate interlocking point. Sample height at the gyration level of the resultant interlocking points indicated higher energy requirement for coarser samples (difficult to compact) (Vavrik, 2000). The effect of compaction level for S5-G2, S6-G2 and S7-G2 with similar binder contents and gradations were 40,80 and 40 gyrations, respectively. The required compaction level of the mixtures could not be observed due to the inconsistency of aggregate packing at 40 gyrations compaction. However, the proposed analytical method gave almost the same value (average 54) for three samples. Similarly, according to both methods the interlocking point variation with binder content showed an increment for higher binder contents.

According to the comparison, interlocking points for all samples were around 50 gyrations with method (b). 
Additionally, percentage $\mathrm{G}_{\mathrm{mm}}$ achieved at the interlocking point was more than $85 \%$ for all except 40 gyration samples. This compaction level has been recommended by most of the specifications and researchers as the suitable compaction for OGFC mix designs using SGC to simulate field compaction (Kandhal \& Mallick, 1999; Watson et al., 2002; Alvarez et al., 2007; NCAT, 2000).

Except gradation and binder content, factors such as compaction method, compaction temperature, rheological properties of binder etc. (Sefidmazgi, 2011) are important and should be controlled to achieve optimum compaction, specially for OGFC to avoid effects on functionality of the pavement in long term service.

\section{CONCLUSION}

A method was developed analytically to identify the interlocking point by using gyrations versus sample height curve (direct measurement of the SGC). Although it was not much sensitive to individual compaction variations, theoretically, method (b) provided more reliable values. According to the results, binder content was highly sensitive to aggregate interlocking than gradation and compaction levels under the proposed method. However, all samples generally achieved optimum compaction around 50 gyrations. As the densification rate is insignificant after the locking and internal aggregate structure and air void content are important in the context of permeability of OGFC, it is essential to identify the optimum compaction requirement for field practicing.

\section{REFERENCES}

Alvarez A.E., Martin A.E., Estakhri C.K., Button J.W., Kraus Z., Prapaitrakul N. \& Glover C.J. (2007). Evaluation and Recommended Improvements for Mix Design of Permeable Friction Courses, Report No. FHWA/TX-08/0-5262-3. Texas Transportation Institute, Texas, USA.

Birgisson B., Roque R., Varadhan A., Tai T. \& Jaiswal L. (2006). Evaluation of Thick Open Graded and Bounded Friction Courses for Florida. Florida Department of Transportation, Florida, USA.

Chandra S. \& Kumar R. (2016). A comparative study on PFC mixes, Proceedings of the $12^{\text {th }}$ International Conference on Transportation Planning and Implementation Methodologies for Developing Countries, 19-21 December, Mumbai, India.

Choubane B., Page G.C. \& Musselman J.A. (1997). Investigation of Water Permeability of Coarse Graded Superpave Pavements. Research Report FL/DOT/SMO/97-
416. State Materials Office, Florida, USA.

Cindy K.E., Alvarez A.E. \& Martin A.E. (2007). Guidelines on Construction and Maintenance of Porous Friction Courses in Texas. Texas Transportation Institute, Texas, USA.

Gunaratne M. \& Pernia Y.M.D. (2015). Automated Determination of the Optimum Binder Content of OpenGraded Friction Course Mixtures using Digital Image Processing. Florida Department of Transportation, Florida, USA.

Gyratory Compactor (operating manual), Available at: http:// www.epc.com.hk/uploadfiles/Gyratory\%20Compactor.pdf, Accessed 10 April 2017.

Kandhal P.S. \& Mallick R.B. (1999). Design of NewGeneration Open-Graded Friction Course, NCAT Report 99-03. National Center for Asphalt Technology, Alabama, USA.

Liu K.W., Alvarez A.E., Martin A.E., Dossey T., Smit A. \& Cindy K.E. (2010). Synthesis of Current Research on Permeable Friction Courses: Performance, Design, Construction, and Maintenance, Report No. FHWA/TX10/0-5836-1. Texas Department of Transportation, Texas, USA.

Liu M., Haung X. \& Xue G. (2016). Effect of double layer porous asphalt pavement of urban streets on noise reduction. International Journal of Sustainable Built Environment 5 (1): 183-196.

DOI: https://doi.org/10.1016/j.ijsbe.2016.02.001

Mahboub K.C. (2004). An introduction to superpave. In: Pavement Analysis and Design (ed. Y.H. Haung), $2^{\text {nd }}$ edition, pp. 682-693. Pearson Education, London, UK.

National Center for Asphalt Technology (NCAT) (2000). Design, Construction and Performance of New Generation Open-Graded Friction Course Mixes, Research synopsisNCAT report 00-01. National Center for Asphalt Technology, Auburn University, USA.

Putman B.J. (2012). Evaluation of Open-Graded Friction Courses: Construction, Maintenance, and Performance. Report No. FHWA-SC-12-04, Clemson University, USA.

Sefidmazgi N.R. (2011). Defining effective aggregate skeleton in asphalt mixture using digital imaging. MSc thesis, University of Wisconsin, Madison, USA.

Smit A.D.F. \& Prozzi J.A. (2013). Quantification of the reduction of wet weather accidents using porous friction courses (PFC), $13^{\text {th }}$ COTA International Conference of Transportation Professionals, University of Texas, USA. DOI: https://doi.org/10.1016/j.sbspro.2013.08.308

Techbrief (2010). Superpave Gyratory Compactors, FHWAHIF-11-032. Federal Highway Administration, Department of Transportation, USA.

Techbrief (2015). Porous Asphalt Pavements with Stone Reservoirs, FHWA-HIF-15-009. Office of Asset Management, Pavements, and Construction, USA.

Vavrik W.R. (2000). Asphalt Mixture Design Concepts to Develop Aggregate Interlocking, PhD thesis, Graduate College of the University of Illinois, Illinois, USA.

Washington Department of Transportation (WDOT) (2016). 
Preparing Hot-Mix Asphalt (HMA) Specimens by Means of the Superpave Gyratory Compactor, Materials Manual M 46-01.24, Washington Department of Transportation, Washington Dc, USA.
Watson D.E., Moore K.A., Williams K. \& Cooley L.A. (2002). Refinement of New Generation Open-Graded Friction Course Mix Design. National Center for Asphalt Technology, USA. 